\title{
CONVERGENCE OF A NEUMANN-DIRICHLET ALGORITHM FOR TWO-BODY CONTACT PROBLEMS WITH NON LOCAL COULOMB'S FRICTION LAW
}

\author{
Guy Bayada $^{1}$, Jalila SABIL ${ }^{2}$ And TAOUfik SAssi ${ }^{3}$
}

\begin{abstract}
In this paper, the convergence of a Neumann-Dirichlet algorithm to approximate Coulomb's contact problem between two elastic bodies is proved in a continuous setting. In this algorithm, the natural interface between the two bodies is retained as a decomposition zone.
\end{abstract}

Mathematics Subject Classification. 65N30, 65N55, 65K05.

Received December 23, 2006. Revised September 12, 2007.

\section{INTRODUCTION}

Domain decomposition methods are usually characterized by an artificial splitting of a given domain where a partial differential equation has to be solved. Then, suitable iterations are performed in which a sequence of local problems are considered. Each of these local problems being defined in a subdomain with convenient boundary conditions. One of the potential interest of this approach is that the computation of solutions for the local problems required much less time than the solution for the initial problem and can be made simultaneously by using parallel computers. Moreover various discretizations or various kinds of solvers can be used to deal with each of the local problems. Several methods have been reviewed in $[9,17,19]$.

At first glance, multibody contact problems in elasticity appear to be a natural domain of application for domain decomposition methods. Many numerical procedures have been proposed in the mechanical literature. They are based on standard discretization techniques for partial differential equations in combination with a special implementation of the non-linear contact conditions (see e.g. [5,6,10-13,20]).

However, so far this kind of approach seems to have been neglected in mathematical literature. One of the first mathematical proof of the convergence for contact decomposition algorithm is proposed in [1]. However, the decomposition boundary is artificial and located inside one of bodies.

More recently, a so-called Neumann-Dirichlet algorithm for the solution of frictionless Signorini contact problem has been proposed and studied in the discretized setting in [14-16]. This new algorithm consists

\footnotetext{
Keywords and phrases. Domain decomposition methods, contact problems, convergence.

${ }^{1}$ INSA-LYON, CNRS UMR 5208, UMR 5514 Bâtiment Léonard de Vinci, 21 Av. J. Capelle, 69621 Villeurbanne Cedex, France. guy.bayada@insa-lyon.fr

${ }^{2}$ Université Henri Poincaré, UFR STMP, Faculté des Sciences et Techniques, Laboratoire LEMTA B.P. 239, 54506 Vandøuvreles-Nancy Cedex, France. Jalila.Sabil@lemta.uhp-nancy.fr

3 Laboratoire de Mathématiques Nicolas Oresme, LMNO CNRS UMR 6139, Université de Caen, Bd. Maréchal Juin, 14032 Caen Cedex, France. Taoufik.Sassi@math.unicaen.fr
} 
to solve in each iteration a linear Neumann problem for one body and a unilateral contact problem for the other by using essentially the contact interface as the boundary data transfer. The convergence of this algorithm in the continuous setting has been proved in $[4,8]$. This last approach has been extended to the contact problem with Coulomb friction. The corresponding discretized Neumann-Dirichlet algorithm and several numerical results are given in $[14,16]$.

The aim of the present paper is to prove the convergence of the Neumann-Dirichlet algorithm for contact problem with Coulomb friction in the continuous setting. The main difficulty in this work is linked with the boundary conditions at the contact interface. They are highly non-linear both in the normal direction (unilateral contact conditions) and in the tangential one (non-differential Coulomb law). A fixed point relaxed procedure is defined for the stresses on the contact surface. For sufficiently small friction coefficient, the convergence of the Neumann-Dirichlet algorithm is proved for the continuous problem. It is also shown, by some numerical calculations, that an optimal relaxation parameter exists and its value is nearly independent of the friction coefficient, the mesh size and of the Young modulus.

The paper is organized as follows:

In Sections 2 and 3, we give a statement of the problem and we propose the natural Neumann-Dirichlet algorithm in the strong formulation. The precise variational formulation of the algorithm is given in Section 4 . We then prove in Section 5, under some assumptions concerning the data, the convergence of the stresses on the contact surface, which in turn proves the convergence of the whole algorithm. In the last, we present some numerical results asserting the efficiency of our algorithm.

\section{Stating the Problem}

Let us consider two elastic bodies, occupying two bounded domains $\Omega^{\alpha}, \alpha=1,2$, of the space $\mathbb{R}^{2}$. The boundary $\Gamma^{\alpha}=\partial \Omega^{\alpha}$ is assumed piecewise continuous, and composed of three complementary non empty parts $\Gamma_{u}^{\alpha}, \Gamma_{l}^{\alpha}$ and $\Gamma_{c}^{\alpha}$ such that $\bar{\Gamma}_{u}^{\alpha} \cap \bar{\Gamma}_{c}^{\alpha}=\emptyset$. Each body $\bar{\Omega}^{\alpha}$ is fixed on the part $\Gamma_{u}^{\alpha}$ and subjected to surface traction forces $\phi^{\alpha}$ in $\left(L^{2}\left(\Gamma_{l}^{\alpha}\right)\right)^{2}$. The body forces are denoted by $\boldsymbol{f}^{\alpha}$ in $\left(L^{2}\left(\Omega^{\alpha}\right)\right)^{2}$. In the initial configuration, both bodies have a common contact portion $\Gamma_{c}=\Gamma_{c}^{1}=\Gamma_{c}^{2}$. In other words, we consider the case when contact zone cannot grow during the deformation process. Unilateral contact with non local Coulomb's friction can take place along the boundary $\Gamma_{c}$. The problem consists in finding the displacement field $\boldsymbol{u}=\left(\boldsymbol{u}^{1}, \boldsymbol{u}^{2}\right)($ where the notation $\boldsymbol{u}^{\alpha}$ stands for $\left.\left.\boldsymbol{u}\right|_{\Omega^{\alpha}}\right)$ and the stress tensor field $\sigma=\left(\sigma\left(\boldsymbol{u}^{1}\right), \sigma\left(\boldsymbol{u}^{2}\right)\right)$ such that: for $\alpha=1,2$

$$
\left\{\begin{array}{lll}
\operatorname{div} \sigma\left(\boldsymbol{u}^{\alpha}\right)+\boldsymbol{f}^{\alpha} & = & \\
\sigma\left(\boldsymbol{u}^{\alpha}\right) \boldsymbol{n}^{\alpha} & =\boldsymbol{\phi}^{\alpha} & \text { on } \Omega_{l}^{\alpha}, \\
\boldsymbol{u}^{\alpha} & =\mathbf{0} & \text { on } \Gamma_{u}^{\alpha},
\end{array}\right.
$$

where the symbol div denotes the divergence operator of a tensor function and is defined as

$$
\operatorname{div} \sigma=\left(\frac{\partial \sigma_{i j}}{\partial x_{j}}\right)_{i}
$$

The summation convention of repeated index is adopted. The elastic constitutive law is given by Hooke's law for homogeneous and isotropic solid:

$$
\sigma_{i j}\left(\boldsymbol{u}^{\alpha}\right)=\mathrm{A}_{i j k h}^{\alpha} e_{k h}\left(\boldsymbol{u}^{\alpha}\right), \quad e\left(\boldsymbol{u}^{\alpha}\right)=\frac{1}{2}\left(\nabla \boldsymbol{u}^{\alpha}+\left(\nabla \boldsymbol{u}^{\alpha}\right)^{T}\right)
$$

where $\mathrm{A}^{\alpha}(x)=\left(a_{i j k h}^{\alpha}(x)\right)_{1 \leq i, j, k, h \leq 2} \in\left(L^{\infty}\left(\Omega^{\alpha}\right)\right)^{16}$ is a fourth-order tensor satisfying the usual symmetry and ellipticity conditions in elasticity, and $e\left(\boldsymbol{u}^{\alpha}\right)$ is the strain tensor. 
We will use the usual notations for the normal and tangential components of the displacement and stress vector on $\Gamma_{c}$

$$
\begin{aligned}
\boldsymbol{u}_{N}^{\alpha} & =\boldsymbol{u}_{i}^{\alpha} \boldsymbol{n}_{i}^{\alpha}, & \boldsymbol{u}_{T_{i}}^{\alpha} & =\boldsymbol{u}_{i}^{\alpha}-\boldsymbol{u}_{N}^{\alpha} \boldsymbol{n}_{i}^{\alpha} \\
\sigma_{N}^{\alpha} & =\sigma_{i j}\left(\boldsymbol{u}^{\alpha}\right) \boldsymbol{n}_{i}^{\alpha} \boldsymbol{n}_{j}^{\alpha}, & \sigma_{T i}^{\alpha} & =\sigma_{i j}\left(\boldsymbol{u}^{\alpha}\right) \boldsymbol{n}_{j}^{\alpha}-\sigma_{N}^{\alpha} \boldsymbol{n}^{\alpha},
\end{aligned}
$$

in which we have denoted by $\boldsymbol{n}^{\alpha}$ the outward normal unit vector to the boundary.

On the interface $\Gamma_{c}$, the unilateral contact law is described by

$$
\begin{gathered}
\sigma_{N}^{1}=\sigma_{N}^{2}=\sigma_{N}, \quad \sigma_{T}^{1}=\sigma_{T}^{2}=\sigma_{T}, \\
{\left[\boldsymbol{u}_{N}\right] \leq 0, \quad \sigma_{N} \leq 0, \quad \sigma_{N}\left[\boldsymbol{u}_{N}\right]=0,}
\end{gathered}
$$

where $\left[\boldsymbol{v}_{N}\right]=\boldsymbol{v}^{1} \cdot \boldsymbol{n}^{1}+\boldsymbol{v}^{2} \cdot \boldsymbol{n}^{2}$, is the jump across the interface of any function $\boldsymbol{v}$ defined on $\Omega^{\alpha}$.

The Coulomb's law with non-local friction is given by

$$
\left\{\begin{array}{l}
\left|\sigma_{T}\right| \leq k\left|S\left(\sigma_{N}\right)\right| \\
\left|\sigma_{T}\right|<k\left|S\left(\sigma_{N}\right)\right| \Longrightarrow\left[\boldsymbol{u}_{T}\right]=0 \\
\left|\sigma_{T}\right|=k\left|S\left(\sigma_{N}\right)\right| \Longrightarrow \exists \nu \geq 0 \quad\left[\boldsymbol{u}_{T}\right]=-\nu \sigma_{T}
\end{array}\right.
$$

where $k(x)$ is the coefficient of friction on the interface $\Gamma_{c}: k(x) \in L^{\infty}\left(\Gamma_{c}\right), k(x) \geq 0$ a.e. on $\Gamma_{c} ; S$ is a regularization operator from the dual of $H^{\frac{1}{2}}\left(\Gamma_{c}\right)$ into $L^{2}\left(\Gamma_{c}\right)$.

It's easy to prove (see for example [7]) that (2.5) is equivalent to:

$$
\left\{\begin{array}{l}
\left|\sigma_{T}\right| \leq k\left|S\left(\sigma_{N}\right)\right| \\
k\left|S\left(\sigma_{N}\right)\right|\left|\left[\boldsymbol{u}_{T}\right]\right|+\sigma_{T}\left[\boldsymbol{u}_{T}\right]=0
\end{array}\right.
$$

\section{Neumann-Dirichlet Algorithm}

Let $0<\theta<1$ be a parameter that will be determined in order to ensure the convergence of the algorithm. Let $g_{0}^{2}$ be a given element on $\Gamma_{c}$. For $i \geq 1$, the sequence of functions $\left(\boldsymbol{u}_{i}^{1}\right)_{i \geq 0}$ and $\left(\boldsymbol{u}_{i}^{2}\right)_{i \geq 0}$ is defined by solving the following problems:

$$
\left\{\begin{array}{lll}
(I) \quad & \operatorname{div} \sigma\left(\boldsymbol{u}_{i}^{2}\right)=\boldsymbol{f}^{2} & \text { in } \Omega^{2} \\
& \sigma\left(\boldsymbol{u}_{i}^{2}\right) \boldsymbol{n}^{2}=\boldsymbol{\phi}^{2} & \text { on } \Gamma_{l}^{2} \\
& \boldsymbol{u}_{i}^{2}=\mathbf{0} & \text { on } \Gamma_{u}^{2} \\
& \sigma\left(\boldsymbol{u}_{i}^{2}\right) \boldsymbol{n}^{2}=g_{i-1}^{2} & \text { on } \Gamma_{c} \\
(I I) \quad & \operatorname{div} \sigma\left(\boldsymbol{u}_{i}^{1}\right)=\boldsymbol{f}^{1} & \text { in } \Omega^{1} \\
& \sigma\left(\boldsymbol{u}_{i}^{1}\right) \boldsymbol{n}^{1}=\boldsymbol{\phi}^{1} & \text { on } \Gamma_{l}^{1} \\
& \boldsymbol{u}_{i}^{1}=\mathbf{0} & \text { on } \Gamma_{u}^{1} \\
& \boldsymbol{u}_{i}^{1} \boldsymbol{n}^{1} \leq-\boldsymbol{u}_{i}^{2} \boldsymbol{n}^{2}, \quad \sigma_{i N}^{1} \leq 0, \quad \sigma_{i_{N}}^{1}\left[\boldsymbol{u}_{i_{N}}\right]=0 & \text { on } \Gamma_{c} \\
& \left|\sigma_{i_{T}}^{1}\right| \leq k\left|S\left(\sigma_{i_{N}}^{2}\right)\right| & \\
& \sigma_{i_{T}}^{1}\left[\boldsymbol{u}_{i_{T}}\right]+k\left|S\left(\sigma_{i_{N}}^{2}\right)\right|\left|\left[\boldsymbol{u}_{i_{T}}\right]\right|=0 & \text { on } \Gamma_{c} \\
(I I I) & g_{i}^{2}:=\theta \sigma_{i}^{1} \boldsymbol{n}^{1}+(1-\theta) g_{i-1}^{2} & \text { on } \Gamma_{c} .
\end{array}\right.
$$

The variational form of this algorithm will be given in (4.9). The proof of its convergence towards the solution of the problem (2.1)-(2.6) will be given in Theorem 5.9. 


\section{THE VARIATIONAL FORMULATION}

In order to study the previous algorithm, let us first define its variational formulation by introducing the following functional spaces:

For $\alpha=1,2$ :

$V^{\alpha}=\left\{\boldsymbol{v}^{\alpha} \in\left(H^{1}\left(\Omega^{\alpha}\right)\right)^{2}, \boldsymbol{v}^{\alpha}=0\right.$ on $\left.\Gamma_{u}^{\alpha}\right\}$, endowed with the norm $\|\cdot\|_{\alpha}=\|\cdot\|_{\left(H^{1}\left(\Omega^{\alpha}\right)\right)^{2}}$.

$V=V^{1} \times V^{2} \quad$ provided with the norm $\|\cdot\|_{V}=\left(\sum_{\alpha=1}^{2}\|\cdot\|_{\alpha}^{2}\right)^{\frac{1}{2}}$.

$K=\left\{\boldsymbol{v} \in V,\left[\boldsymbol{v}_{N}\right] \leq 0\right.$ a.e. on $\left.\Gamma_{c}\right\}$.

$\mathcal{H}^{\frac{1}{2}}\left(\Gamma_{c}\right)=\left\{\boldsymbol{\varphi} \in\left(L^{2}\left(\Gamma_{c}\right)\right)^{2} ; \exists \boldsymbol{v} \in V^{\alpha} ; \alpha=1,2 ; \gamma \boldsymbol{v}_{\mid \Gamma_{c}}=\varphi\right\}$, provided with the norm

$$
\|\varphi\|_{\frac{1}{2}, \Gamma_{c}}=\inf \left\{\|\boldsymbol{v}\|_{\alpha} ; \boldsymbol{v} \in V^{\alpha}, \gamma \boldsymbol{v}_{\mid \Gamma_{c}}=\varphi\right\},
$$

where $\gamma$ is the usual trace operator.

As $\Gamma_{c}^{\alpha} \cap \Gamma_{u}^{\alpha}=\emptyset$, the space $\mathcal{H}^{\frac{1}{2}}\left(\Gamma_{c}\right)$ does not depend on $\alpha$.

Let us denote by $V^{\alpha^{\prime}}$ the dual space of $V^{\alpha}$ and by $\langle\cdot, \cdot\rangle_{\alpha}$ the duality pairing of the two spaces. The dual norm on $V^{\alpha^{\prime}}$ is given by:

$$
\|\psi\|_{V^{\alpha^{\prime}}}=\sup _{v \in V^{\alpha}} \frac{\left|\langle\psi, \boldsymbol{v}\rangle_{\alpha}\right|}{\|\boldsymbol{v}\|_{\alpha}}
$$

Let us introduce for all $\varphi$ in $H^{\frac{1}{2}}\left(\Gamma_{c}\right)$ the set:

$$
V_{-}^{1}(\varphi)=\left\{\boldsymbol{v}^{1} \in V^{1}, \boldsymbol{v}^{1} \cdot \boldsymbol{n}^{1} \leq-\varphi \text { a.e on } \Gamma_{c}\right\} .
$$

We will denote by:

$$
\begin{aligned}
a^{\alpha}(\boldsymbol{u}, \boldsymbol{v}) & =\int_{\Omega^{\alpha}} \mathrm{A}_{i j k l}^{\alpha} e_{i j}(\boldsymbol{u}) e_{k l}(\boldsymbol{v}) \mathrm{d} x, \quad a(\boldsymbol{u}, \boldsymbol{v})=\sum_{\alpha=1}^{2} a^{\alpha}(\boldsymbol{u}, \boldsymbol{v}), \\
L^{\alpha}(\boldsymbol{v}) & =\int_{\Omega^{\alpha}} \boldsymbol{f}^{\alpha} \cdot \boldsymbol{v} \mathrm{d} x+\int_{\Gamma_{l}^{\alpha}} \boldsymbol{\phi}^{\alpha} \cdot \boldsymbol{v} \mathrm{d} x, \quad L(\boldsymbol{v})=\sum_{\alpha=1}^{2} L^{\alpha}(\boldsymbol{v}) .
\end{aligned}
$$

The bilinear form $a^{\alpha}(.,$.$) is continuous and coercive since \operatorname{mes}\left(\Gamma_{u}^{\alpha}\right)>0$, so $a^{\alpha}(.,$.$) satisfies:$

$$
\begin{aligned}
& \exists M^{\alpha}>0, \quad\left|a^{\alpha}(\boldsymbol{u}, \boldsymbol{v})\right| \leq M^{\alpha}\|\boldsymbol{u}\|_{\alpha}\|\boldsymbol{v}\|_{\alpha} \quad \forall \boldsymbol{u}, \boldsymbol{v} \in V^{\alpha}, \\
& \exists m^{\alpha}>0, \quad a^{\alpha}(\boldsymbol{v}, \boldsymbol{v}) \geq m^{\alpha}\|\boldsymbol{v}\|_{\alpha}^{2} \quad \forall \boldsymbol{v} \in V^{\alpha} .
\end{aligned}
$$

Let

$$
M=\sum_{\alpha=1}^{2} M^{\alpha}, \quad m=\min _{\alpha=1,2} m^{\alpha},
$$

so

$$
\begin{aligned}
& |a(\boldsymbol{u}, \boldsymbol{v})| \leq M\|\boldsymbol{u}\|_{V}\|\boldsymbol{v}\|_{V}, \quad \forall \boldsymbol{u}, \boldsymbol{v} \in V, \\
& a(\boldsymbol{v}, \boldsymbol{v}) \geq m\|\boldsymbol{v}\|_{V}^{2} \quad \forall \boldsymbol{v} \in V .
\end{aligned}
$$


Let us first recall that the variational formulation of the initial problem (2.1)-(2.6) is given by:

$$
\left(P_{1}\right)\left\{\begin{array}{l}
\text { Find } \boldsymbol{u} \text { in } K \text { such that: } \\
a(\boldsymbol{u}, \boldsymbol{v}-\boldsymbol{u})+\int_{\Gamma_{c}} k\left|S\left(\sigma_{N}\right)\right|\left(\left|\left[\boldsymbol{v}_{T}\right]\right|-\left|\left[\boldsymbol{u}_{T}\right]\right|\right) \geq L(\boldsymbol{v}-\boldsymbol{u}) \quad \forall \boldsymbol{v} \in K .
\end{array}\right.
$$

which has at least one solution and this solution is unique for a sufficiently small $\|k\|_{L^{\infty}\left(\Gamma_{c}\right)}$ (see e.g. [7]).

In the following, we will use some lift operators which allow us to build specific functions in $\Omega^{\alpha}$ from their values on $\Gamma_{c}$.

For $\alpha=1,2$, let

$$
\begin{gathered}
\boldsymbol{R}_{D}^{\alpha}: \mathcal{H}^{\frac{1}{2}}\left(\Gamma_{c}\right) \longrightarrow V^{\alpha} \\
\boldsymbol{\varphi} \longrightarrow \boldsymbol{R}_{D}^{\alpha} \boldsymbol{\varphi} . \\
\left\{\begin{array}{lc}
a^{\alpha}\left(\boldsymbol{R}_{D}^{\alpha} \boldsymbol{\varphi}, \boldsymbol{v}\right)=0 & \forall \boldsymbol{v} \in V^{\alpha} \text { such that } \gamma \boldsymbol{v}=0 \text { on } \Gamma_{c} \\
\gamma\left(\boldsymbol{R}_{D}^{\alpha} \boldsymbol{\varphi}\right)=\boldsymbol{\varphi} & \text { on } \Gamma_{c},
\end{array}\right.
\end{gathered}
$$

whose strong formulation is given by:$$
\begin{cases}\operatorname{div} \sigma^{\alpha}=0 \text { in } \Omega^{\alpha}, & \text { with } A^{\alpha} e\left(\boldsymbol{R}_{D}^{\alpha} \boldsymbol{\varphi}\right)=\sigma^{\alpha} \\ \gamma\left(\boldsymbol{R}_{D}^{\alpha} \boldsymbol{\varphi}\right)=\boldsymbol{\varphi} & \text { on } \Gamma_{c} \\ \sigma^{\alpha} \boldsymbol{n}^{\alpha}=0 & \text { on } \Gamma_{l}^{\alpha} \\ \gamma\left(\boldsymbol{R}_{D}^{\alpha} \boldsymbol{\varphi}\right)=0 & \text { on } \Gamma_{u}^{\alpha} .\end{cases}
$$

\section{Proposition 4.1.}

(i) The three norms $\|\varphi\|_{\frac{1}{2}, \Gamma_{c}}$ and $\left\|\boldsymbol{R}_{D}^{\alpha} \boldsymbol{\varphi}\right\|_{\alpha}, \alpha=1,2$ are equivalent on $\mathcal{H}^{\frac{1}{2}}\left(\Gamma_{c}\right)$.

(ii) There exists a constant $C>0$ such that

$$
\forall v \in V^{\alpha}\left\|\boldsymbol{R}_{D}^{\beta} \gamma \boldsymbol{v}_{\mid \Gamma_{c}}\right\|_{\beta} \leq C\|\boldsymbol{v}\|_{\alpha} \quad \alpha, \beta=1,2 \text { and } \alpha \neq \beta
$$

Proof. (i) Using the definition of $\boldsymbol{R}_{D}^{\alpha}$, we have:

$$
\gamma\left(\boldsymbol{R}_{D}^{\alpha}(\varphi)\right)=\boldsymbol{\varphi}
$$

so from the definition of $\|\cdot\|_{\frac{1}{2}, \Gamma_{c}}$, we gain

$$
\|\varphi\|_{\frac{1}{2}, \Gamma_{c}} \leq\left\|\boldsymbol{R}_{D}^{\alpha} \boldsymbol{\varphi}\right\|_{1} .
$$

Conversely, let us consider the range of $\mathcal{H}^{\frac{1}{2}}\left(\Gamma_{c}\right)$ by $\boldsymbol{R}_{D}^{\alpha}$

$$
W^{\alpha}=\boldsymbol{R}_{D}^{\alpha}\left(\mathcal{H}^{\frac{1}{2}}\left(\Gamma_{c}\right)\right)
$$

As for any $\varphi$ in $\mathcal{H}^{\frac{1}{2}}\left(\Gamma_{c}\right)$, problem (4.5) has a unique solution, and as the trace restriction on $\Gamma_{c}$ of any function in $W^{\alpha}$ is unique, so the application $\boldsymbol{R}_{D}^{\alpha}$ is a bijection from $\mathcal{H}^{\frac{1}{2}}\left(\Gamma_{c}\right)$ in $W^{\alpha}$. Let us consider its inverse $\boldsymbol{R}^{\alpha_{D}^{-1}}$. It is easy to prove that this last bijection is linear, and we can obtain its continuity from (4.7).

Moreover, as $W^{\alpha}$ is a closed sub-space of $V^{\alpha}$ which is a Banach space, so $W^{\alpha}$ is also a Banach one.

Using the homeomorphism Banach theorem (see e.g.[18]), we obtain that $\boldsymbol{R}_{D}^{\alpha}$ is continuous from $\mathcal{H}^{\frac{1}{2}}\left(\Gamma_{c}\right)$ in $W^{\alpha}$, i.e.

$$
\left\|\boldsymbol{R}_{D}^{\alpha} \boldsymbol{\varphi}\right\|_{1} \leq C\|\varphi\|_{\frac{1}{2}, \Gamma_{c}} \quad \forall \varphi \in \mathcal{H}^{\frac{1}{2}}\left(\Gamma_{c}\right) .
$$

Consequently, the three norms $\|\varphi\|_{\frac{1}{2}, \Gamma_{c}}$ and $\left\|\boldsymbol{R}_{D}^{\alpha} \boldsymbol{\varphi}\right\|_{\alpha}, \alpha=1,2$ are equivalent.

(ii) Let $\boldsymbol{v}$ be in $V^{\alpha}$, using inequality (4.8) and the trace theorem, the result is established. 
Remark 4.2. For sake of simplicity, we will write in the following $\boldsymbol{R}_{D}^{\alpha}(\gamma \boldsymbol{v})$ instead of $\boldsymbol{R}_{D}^{\alpha}\left(\gamma \boldsymbol{v}_{\mid \Gamma_{c}}\right)$.

The weak formulation of the algorithm (3.1) is given by:

Let $g_{0}^{2}$ be a given element in $V^{2^{\prime}}$. For $i \geq 1$, let us define the sequence of functions $\left(\boldsymbol{u}_{i}^{1}\right)_{i \geq 0}$ in $V^{1}$ and $\left(\boldsymbol{u}_{i}^{2}\right)_{i \geq 0}$ in $V^{2}$ by solving the following problems:

$$
\left(Q_{i}\right)\left\{\begin{array}{l}
(I) \text { Find } \boldsymbol{u}_{i}^{2} \text { in } V^{2} \text { such that: } \\
a^{2}\left(\boldsymbol{u}_{i}^{2}, \boldsymbol{v}\right)=L^{2}(\boldsymbol{v})+\left\langle g_{i-1}^{2}, \boldsymbol{v}\right\rangle_{2} \forall \boldsymbol{v} \in V^{2} \\
(I I) \text { Find } \boldsymbol{u}_{i}^{1} \text { in } V^{1}\left(\boldsymbol{u}_{i_{N}}^{2}\right) \text { such that: } \\
a^{1}\left(\boldsymbol{u}_{i}^{1}, \boldsymbol{w}+\boldsymbol{R}_{D}^{1}\left(\gamma \boldsymbol{u}_{i}^{2}\right)-\boldsymbol{u}_{i}^{1}\right)+\int_{\Gamma_{c}} k\left|S\left(\sigma_{i_{N}}\left(\boldsymbol{u}_{i}^{2}\right)\right)\right|\left(\left|\boldsymbol{w}_{T}\right|-\left|\left[\boldsymbol{u}_{i_{T}}\right]\right|\right) \\
\quad \geq L^{1}\left(\boldsymbol{w}+\boldsymbol{R}_{D}^{1}\left(\gamma \boldsymbol{u}_{i}^{2}\right)-\boldsymbol{u}_{i}^{1}\right) \forall \boldsymbol{w} \in V_{-}^{1}(0) \\
(I I I)\left\langle g_{i}^{2}, \boldsymbol{v}\right\rangle_{2}:= \\
\theta\left(-a^{1}\left(\boldsymbol{u}_{i}^{1}, \boldsymbol{R}_{D}^{1} \gamma \boldsymbol{v}\right)+L^{1}\left(\boldsymbol{R}_{D}^{1} \gamma \boldsymbol{v}\right)\right)+(1-\theta)\left\langle g_{i-1}^{2}, \boldsymbol{v}\right\rangle_{2} \forall \boldsymbol{v} \in V^{2} .
\end{array}\right.
$$

Remark 4.3. Using inequality (ii) of Proposition 4.1, the expression $\left\langle g_{i}^{2}, \boldsymbol{v}\right\rangle_{2}$ in (4.9)(III) has sense, i.e. $g_{i}^{2}$ belongs to $V^{2^{\prime}}$.

\section{Convergence}

The convergence of the algorithm towards the solution of $\left(P_{1}\right)$ and then of (2.1)-(2.6) is given by a two-step procedure. We prove first, in Proposition 5.1, that the convergence of $\left(g_{i}^{2}\right)_{i}$ in $V^{2^{\prime}}$ induces the strong convergence of $\left(\boldsymbol{u}_{i}^{\alpha}\right)_{i}$. Then, in Proposition 5.6, the contraction of an auxiliary operator $T_{\theta}$ defined by (5.7) is proved. This yields the convergence of the sequence $\left(g_{i}^{2}\right)_{i}$ by way of Theorem 5.9 .

Proposition 5.1. Let us suppose that there exists $0<\theta_{\min }<1$ such that for any $\theta, \theta \geq \theta_{\min }$, the sequence $\left(g_{i}^{2}\right)_{i \geq 0}$ converges in $V^{2}$. Then the sequence $\left(\boldsymbol{u}_{i}^{1}, \boldsymbol{u}_{i}^{2}\right)$ (strongly) converges in $V$ to the solution $\left(\boldsymbol{u}^{1}, \boldsymbol{u}^{2}\right)$ of $\left(P_{1}\right)$.

Proof. Let $\left(\boldsymbol{u}_{i}^{1}, \boldsymbol{u}_{i}^{2}\right)$ and $\left(\boldsymbol{u}_{j}^{1}, \boldsymbol{u}_{j}^{2}\right)$ be solutions of the problems $\left(Q_{i}\right)$ and $\left(Q_{j}\right)$ respectively, so from (4.9)(III) we have:

$$
\begin{gathered}
a^{1}\left(\boldsymbol{u}_{i}^{1}-\boldsymbol{u}_{j}^{1}, \boldsymbol{R}_{D}^{1} \gamma \boldsymbol{v}\right)=-\left\langle g_{i-1}^{2}-g_{j-1}^{2}, \boldsymbol{v}\right\rangle_{2}-\left\langle\frac{g_{i}^{2}-g_{i-1}^{2}}{\theta}-\frac{g_{j}^{2}-g_{j-1}^{2}}{\theta}, \boldsymbol{v}\right\rangle_{2} \\
\forall \boldsymbol{v} \in V^{2}
\end{gathered}
$$

Choosing $\boldsymbol{v}=\boldsymbol{R}_{D}^{2} \gamma\left(\boldsymbol{u}_{i}^{1}-\boldsymbol{u}_{j}^{1}\right)$ in the previous inequality and using the fact that $\boldsymbol{R}_{D}^{1} \gamma\left(\boldsymbol{R}_{D}^{2} \gamma\left(\boldsymbol{u}_{i}^{1}-\boldsymbol{u}_{j}^{1}\right)\right)=\boldsymbol{u}_{i}^{1}-\boldsymbol{u}_{j}^{1}$, we get:

$$
\left\|\boldsymbol{u}_{i}^{1}-\boldsymbol{u}_{j}^{1}\right\|_{1}^{2} \leq \frac{1}{\theta_{\min } m}\left(\left\|g_{i-1}^{2}-g_{j-1}^{2}\right\|_{V^{2^{\prime}}}+\left\|g_{i}^{2}-g_{i-1}^{2}\right\|_{V^{2^{\prime}}}+\left\|g_{j}^{2}-g_{j-1}^{2}\right\|_{V^{2^{\prime}}}\right)\left\|\boldsymbol{R}_{D}^{2} \gamma\left(\boldsymbol{u}_{i}^{1}-\boldsymbol{u}_{j}^{1}\right)\right\|_{2} .
$$

Using Proposition 4.1(i), and the strong convergence of $\left(g_{i}^{2}\right)_{i \geq 0}$ in $V^{2^{\prime}}$, one obtains the convergence of $\left(\boldsymbol{u}_{i}^{1}\right)_{i}$.

The strong convergence of $\left(\boldsymbol{u}_{i}^{2}\right)_{i}$ can be obtained from the one of $\left(g_{i}^{2}\right)_{i \geq 0}$, by using the coerciveness of $a^{2}(\cdot, \cdot)$ in $(4.9)(I)$. 
Finally, we pass to the limit in equations $(I I)$ and $(I I I)$ of problem $\left(Q_{i}\right)$. The limit of $\left(\boldsymbol{u}_{i}^{\alpha}\right)_{i}, \alpha=1,2$ appears to be a solution of the two following problems:

$$
\begin{aligned}
& \left(P_{2}\right)\left\{\begin{array}{l}
\text { Find } \boldsymbol{u}^{2} \text { in } V^{2} \text { such that: } \\
a^{2}\left(\boldsymbol{u}^{2}, \boldsymbol{v}^{2}\right)=L^{2}\left(\boldsymbol{v}^{2}\right)-a^{1}\left(\boldsymbol{u}^{1}, \boldsymbol{R}_{D}^{1}\left(\gamma \boldsymbol{v}^{2}\right)\right)+L^{1}\left(\boldsymbol{R}_{D}^{1}\left(\gamma \boldsymbol{v}^{2}\right)\right) \quad \forall \boldsymbol{v}^{2} \in V^{2} .
\end{array}\right. \\
& \left(P_{3}^{\prime}\right)\left\{\begin{array}{r}
\text { Find } \boldsymbol{u}^{1} \text { in } V_{-}^{1}\left(\boldsymbol{u}_{N}^{2}\right) \text { such that: } \\
a^{1}\left(\boldsymbol{u}^{1}, \boldsymbol{w}+\boldsymbol{R}_{D}^{1}\left(\gamma \boldsymbol{u}^{2}\right)-\boldsymbol{u}^{1}\right)+\int_{\Gamma_{c}} k\left|S\left(\sigma_{N}\right)\right|\left(\left|\boldsymbol{w}_{T}\right|-\left|\left[\boldsymbol{u}_{T}\right]\right|\right) \\
\geq L^{1}\left(\boldsymbol{w}+\boldsymbol{R}_{D}^{1}\left(\gamma \boldsymbol{u}^{2}\right)-\boldsymbol{u}^{1}\right) \quad \forall \boldsymbol{w} \in V_{-}^{1}(0) .
\end{array}\right.
\end{aligned}
$$

The following Lemma 5.2 proves that problems $\left(P_{2}\right)$ and $\left(P_{3}^{\prime}\right)$ imply $\left(P_{1}\right)$ so allowing us to finish the proof of Proposition 5.1.

Lemma 5.2. Assuming that a solution exists for problems $\left(P_{2}\right)$ and $\left(P_{3}^{\prime}\right)$, then there exists also a solution for problem $\left(P_{1}\right)(4.4)$.

Proof. Let us make the following shift for the test function $\boldsymbol{w}$ in $\left(P_{3}^{\prime}\right): \boldsymbol{v}^{1}=\boldsymbol{w}+\boldsymbol{R}_{D}^{1}\left(\gamma \boldsymbol{u}^{2}\right)$. We obtain the following problem $\left(P_{3}\right)$ which is equivalent to problem $\left(P_{3}^{\prime}\right)$ :

$$
\left(P_{3}\right)\left\{\begin{aligned}
\text { Find } \boldsymbol{u}^{1} \text { in } V_{-}^{1}\left(\boldsymbol{u}_{N}^{2}\right) & \text { such that: } \\
a^{1}\left(\boldsymbol{u}^{1}, \boldsymbol{v}^{1}-\boldsymbol{u}^{1}\right)+\int_{\Gamma_{c}} k\left|S\left(\sigma_{N}\right)\right|\left(\left|\boldsymbol{v}_{T}^{1}-\boldsymbol{u}_{T}^{2}\right|-\left|\left[\boldsymbol{u}_{T}\right]\right|\right) & \geq L^{1}\left(\boldsymbol{v}^{1}-\boldsymbol{u}^{1}\right) \\
& \forall \boldsymbol{v}^{1} \in V_{-}^{1}\left(\boldsymbol{u}_{N}^{2}\right) .
\end{aligned}\right.
$$

Let $\boldsymbol{v}=\left(\boldsymbol{v}^{1}, \boldsymbol{v}^{2}\right)$ in $K$, as $\boldsymbol{v}^{1} \cdot \boldsymbol{n}^{1}+\left(\boldsymbol{v}^{2}-\boldsymbol{u}^{2}\right) \cdot \boldsymbol{n}^{2}=\left[\boldsymbol{v}_{N}\right]-\boldsymbol{u}_{N}^{2} \leq-\boldsymbol{u}_{N}^{2}$ on $\Gamma_{c}$, so $\boldsymbol{w}=\boldsymbol{v}^{1}-\boldsymbol{R}_{D}^{1} \gamma\left(\boldsymbol{v}^{2}-\boldsymbol{u}^{2}\right) \in V_{-}^{1}\left(\boldsymbol{u}_{N}^{2}\right)$. Choosing now $w$ (resp. $\boldsymbol{v}^{2}-\boldsymbol{u}^{2}$ ) as test function in $\left(P_{3}\right)$ (resp. in $\left(P_{2}\right)$ ), $\left(P_{1}\right)$ is obtained by addition.

In order to prove the convergence of $\left(g_{n}^{2}\right)_{n}$, let us introduce the operator $T$ defined by:

$$
\begin{aligned}
T: V^{2^{\prime}} & \longrightarrow V^{2^{\prime}} \\
\psi & \longmapsto T \psi
\end{aligned}
$$

such that:

$$
\langle T \psi, \boldsymbol{v}\rangle_{2}=-a^{1}\left(\boldsymbol{w}^{1}, \boldsymbol{R}_{D}^{1} \gamma \boldsymbol{v}\right)+L^{1}\left(\boldsymbol{R}_{D}^{1} \gamma \boldsymbol{v}\right) \forall \boldsymbol{v} \in V^{2},
$$

where $\boldsymbol{w}^{1}$ is the solution of the following obstacle problem:

$$
\left\{\begin{array}{c}
\text { Find } \boldsymbol{w}^{1} \text { in } V_{-}^{1}\left(\boldsymbol{w}_{N}^{2}\right) \text { such that: } \\
a^{1}\left(\boldsymbol{w}^{1}, \boldsymbol{v}-\boldsymbol{w}^{1}\right)+\int_{\Gamma_{c}} k\left|S\left(\sigma_{N}\left(\boldsymbol{w}^{2}\right)\right)\right|\left(\left|\boldsymbol{v}_{T}-\boldsymbol{w}_{T}^{2}\right|-\left|\boldsymbol{w}_{T}^{1}-\boldsymbol{w}_{T}^{2}\right|\right) \geq \\
L^{1}\left(\boldsymbol{v}-\boldsymbol{w}^{1}\right) \quad \forall \boldsymbol{v} \in V_{-}^{1}\left(\boldsymbol{w}_{N}^{2}\right)
\end{array}\right.
$$

and $\boldsymbol{w}^{2}$ is the solution of the Neumann problem:

$$
\left\{\begin{array}{l}
\text { Find } \boldsymbol{w}^{2} \text { in } V^{2} \text { such that: } \\
a^{2}\left(\boldsymbol{w}^{2}, \boldsymbol{v}\right)=L^{2}(\boldsymbol{v})+\langle\psi, \boldsymbol{v}\rangle_{2} \quad \forall \boldsymbol{v} \in V^{2}
\end{array}\right.
$$

We also define the operator $T_{\theta}$ by:

$$
\begin{aligned}
T_{\theta}: V^{2^{\prime}} & \longrightarrow V^{2^{\prime}} \\
\psi & \longmapsto T_{\theta}(\psi)=\theta T(\psi)+(1-\theta) \psi
\end{aligned}
$$


We shall denote by:

$C_{R}$ : the maximum of the constants of continuity for $\boldsymbol{R}_{D}^{1}$ and $\boldsymbol{R}_{D}^{2}$.

$C_{s}$ : the maximum with respect of $\alpha=1,2$ of the constant of continuity of the application $S\left(\sigma_{N}^{\alpha}(\cdot)\right)$ which to each $\boldsymbol{w}^{\alpha}$ in $V^{\alpha}$ associates $S\left(\sigma_{N}^{\alpha}\left(\boldsymbol{w}^{\alpha}\right)\right)$, the regularization of $\sigma_{N}^{\alpha}\left(\boldsymbol{w}^{\alpha}\right)$ in $L^{2}\left(\Gamma_{c}\right)$.

$C_{t r}$ : the maximum with respect of $\alpha=1,2$ of the constants of continuity of both following trace applications:

$$
\operatorname{Tr}^{\alpha}: V^{\alpha} \longrightarrow \mathcal{H}^{\frac{1}{2}}\left(\Gamma_{c}\right), \quad \boldsymbol{v} \longmapsto \boldsymbol{v}_{T}
$$

where $\boldsymbol{v}_{T}$ is the tangential component of $\boldsymbol{v}$.

$C_{K}=\|k\|_{L^{\infty}\left(\Gamma_{c}\right)} C_{s} C_{t r}$, where $k$ is the friction coefficient introduced in (2.6).

Lemma 5.3. For $\boldsymbol{w}^{2}$ and $\tilde{\boldsymbol{w}}^{2}$ in $V^{2}$, let $\boldsymbol{w}^{1}$ and $\tilde{\boldsymbol{w}}^{1}$ be solutions of the problems:

$$
\begin{aligned}
& \left\{\begin{array}{c}
\text { Find } \boldsymbol{w}^{1} \text { in } V_{-}^{1}\left(\boldsymbol{w}_{N}^{2}\right) \text { such that: } \\
a^{1}\left(\boldsymbol{w}^{1}, \boldsymbol{v}-\boldsymbol{w}^{1}\right)+\int_{\Gamma_{c}} k\left|S\left(\sigma_{N}\left(\boldsymbol{w}^{2}\right)\right)\right|\left(\left|\boldsymbol{v}_{T}-\boldsymbol{w}_{T}^{2}\right|-\left|\left[\boldsymbol{w}_{T}\right]\right|\right) \geq \\
L^{1}\left(\boldsymbol{v}-\boldsymbol{w}^{1}\right) \quad \forall \boldsymbol{v} \in V_{-}^{1}\left(\boldsymbol{w}_{N}^{2}\right) .
\end{array}\right. \\
& \left\{\begin{array}{c}
\text { Find } \tilde{\boldsymbol{w}}^{1} \text { in } V_{-}^{1}\left(\tilde{\boldsymbol{w}}_{N}^{2}\right) \text { such that: } \\
a^{1}\left(\tilde{\boldsymbol{w}}^{1}, \boldsymbol{v}-\tilde{\boldsymbol{w}}^{1}\right)+\int_{\Gamma_{c}} k\left|S\left(\sigma_{N}\left(\tilde{\boldsymbol{w}}^{2}\right)\right)\right|\left(\left|\boldsymbol{v}_{T}-\tilde{\boldsymbol{w}}_{T}^{2}\right|-\left|\left[\tilde{\boldsymbol{w}}_{T}\right]\right|\right) \geq \\
L^{1}\left(\boldsymbol{v}-\tilde{\boldsymbol{w}}^{1}\right) \quad \forall \boldsymbol{v} \in V_{-}^{1}\left(\tilde{\boldsymbol{w}}_{N}^{2}\right),
\end{array}\right.
\end{aligned}
$$

then the following estimate holds:

$$
\left\|\boldsymbol{w}^{1}-\tilde{\boldsymbol{w}}^{1}\right\|_{1} \leq\left(\frac{\left(M C_{R}+C_{K}\right)}{m}+\sqrt{\frac{C_{K}}{m}}\right)\left\|\boldsymbol{w}^{2}-\tilde{\boldsymbol{w}}^{2}\right\|_{2} .
$$

Proof. We choose $\boldsymbol{v}=\tilde{\boldsymbol{w}}^{1}-\boldsymbol{R}_{D}^{1}\left(\tilde{\boldsymbol{w}}^{2}\right)+\boldsymbol{R}_{D}^{1}\left(\boldsymbol{w}^{2}\right)$ in (5.9) and $\boldsymbol{v}=\boldsymbol{w}^{1}-\boldsymbol{R}_{D}^{1}\left(\boldsymbol{w}^{2}\right)+\boldsymbol{R}_{D}^{1}\left(\tilde{\boldsymbol{w}}^{2}\right)$ in (5.10). By adding both inequalities, we obtain

$$
\begin{aligned}
a^{1}\left(\boldsymbol{w}^{1}-\tilde{\boldsymbol{w}}^{1}, \boldsymbol{w}^{1}-\tilde{\boldsymbol{w}}^{1}\right) \leq a^{1}\left(\boldsymbol{w}^{1}-\tilde{\boldsymbol{w}}^{1}, \boldsymbol{R}_{D}^{1}\left(\boldsymbol{w}^{2}\right)-\boldsymbol{R}_{D}^{1}\left(\tilde{\boldsymbol{w}}^{2}\right)\right) \\
\quad-\int_{\Gamma_{c}} k\left(\left|S\left(\sigma_{N}\left(\boldsymbol{w}^{2}\right)\right)\right|-\left|S\left(\sigma_{N}\left(\tilde{\boldsymbol{w}}^{2}\right)\right)\right|\right)\left(\left|\left[\boldsymbol{w}_{T}\right]\right|-\left|\left[\tilde{\boldsymbol{w}}_{T}\right]\right|\right) .
\end{aligned}
$$

The continuity of the applications $S\left(\sigma_{N}(\cdot)\right)$ and $\operatorname{Tr}^{\alpha}$ defined in (5.8) induces

$$
-\int_{\Gamma_{c}} k\left(\left|S\left(\sigma_{N}\left(\boldsymbol{w}^{2}\right)\right)\right|-\left|S\left(\sigma_{N}\left(\tilde{\boldsymbol{w}}^{2}\right)\right)\right|\right)\left(\left|\left[\boldsymbol{w}_{T}\right]\right|-\left|\left[\tilde{\boldsymbol{w}}_{T}\right]\right|\right) \leq C_{K}\left(\left\|\boldsymbol{w}^{1}-\tilde{\boldsymbol{w}}^{1}\right\|_{1}\left\|\boldsymbol{w}^{2}-\tilde{\boldsymbol{w}}^{2}\right\|_{2}+\left\|\boldsymbol{w}^{2}-\tilde{\boldsymbol{w}}^{2}\right\|_{2}^{2}\right) .
$$

In (5.12), we use for the left-hand side, the coerciveness of $a^{1}(.,$.$) , and for the right-hand side, the continuity$ of the extension $\boldsymbol{R}_{D}^{\alpha}$ and inequality (5.13) to obtain:

$$
m\left\|\boldsymbol{w}^{1}-\tilde{\boldsymbol{w}}^{1}\right\|_{1}^{2} \leq\left(M C_{R}+C_{K}\right)\left\|\boldsymbol{w}^{1}-\tilde{\boldsymbol{w}}^{1}\right\|_{1}\left\|\boldsymbol{w}^{2}-\tilde{\boldsymbol{w}}^{2}\right\|_{2}+C_{K}\left\|\boldsymbol{w}^{2}-\tilde{\boldsymbol{w}}^{2}\right\|_{2}^{2},
$$

hence

$$
m\left(\left\|\boldsymbol{w}^{1}-\tilde{\boldsymbol{w}}^{1}\right\|_{1}-\frac{M C_{R}+C_{K}}{2 m}\left\|\boldsymbol{w}^{2}-\tilde{\boldsymbol{w}}^{2}\right\|_{2}\right)^{2} \leq\left(\frac{\left(M C_{R}+C_{K}\right)^{2}}{4 m}+C_{K}\right)\left\|\boldsymbol{w}^{2}-\tilde{\boldsymbol{w}}^{2}\right\|_{2}^{2} .
$$

Then the required result is obtained. 
Proposition 5.4. The operator $T$ is Lipschitz continuous:

$\forall \psi, \tilde{\psi}$ in $V^{2^{\prime}} \quad\|T \psi-T \tilde{\psi}\|_{V^{2^{\prime}}} \leq C_{T}\|\psi-\tilde{\psi}\|_{V^{2^{\prime}}}$ with

$$
C_{T}=\frac{M C_{R}}{m^{2}}\left(M C_{R}+C_{K}+\sqrt{C_{K} m}\right) .
$$

Proof. Let $\psi$ and $\tilde{\psi}$ in $V^{2}, \boldsymbol{w}^{1}$ (resp. $\tilde{\boldsymbol{w}}^{1}$ ) and $\boldsymbol{w}^{2}$ (resp. $\tilde{\boldsymbol{w}}^{2}$ ) be the solutions of (5.5) and (5.6), so

$$
\langle T \psi-T \tilde{\psi}, \boldsymbol{v}\rangle_{2}=-a^{1}\left(\boldsymbol{w}^{1}-\tilde{\boldsymbol{w}}^{1}, \boldsymbol{R}_{D}^{1}(\gamma v)\right) \quad \forall \boldsymbol{v} \in V^{2} .
$$

From the continuity of $a^{1}(\cdot, \cdot)$ and Proposition $4.1(i)$, we get:

$$
\|T \psi-T \tilde{\psi}\|_{V^{2^{\prime}}} \leq M C_{R}\left\|\boldsymbol{w}^{1}-\tilde{\boldsymbol{w}}^{1}\right\|_{1}
$$

According to (5.6), one has

$$
a^{2}\left(\boldsymbol{w}^{2}-\tilde{\boldsymbol{w}}^{2}, \boldsymbol{v}\right)=\langle\psi-\tilde{\psi}, \boldsymbol{v}\rangle_{2} \quad \forall \boldsymbol{v} \in V^{2} .
$$

The coerciveness of $a^{2}(\cdot, \cdot)$ implies

$$
\left\|\boldsymbol{w}^{2}-\tilde{\boldsymbol{w}}^{2}\right\|_{2} \leq \frac{1}{m}\|\psi-\tilde{\psi}\|_{V^{2^{\prime}}}
$$

From (5.18), (5.11) and (5.19), we get the existence of a constant $C_{T}$ such that:

$$
\|T(\psi)-T(\tilde{\psi})\|_{V^{2^{\prime}}} \leq C_{T}\|\psi-\tilde{\psi}\|_{V^{2^{\prime}}} .
$$

Let us define the following operator $\boldsymbol{R}^{2}$ :

$$
\begin{aligned}
\boldsymbol{R}^{2}: & V^{2^{\prime}} \\
\psi & \longrightarrow V^{2} \\
\psi & \longmapsto \boldsymbol{R}^{2} \psi
\end{aligned}
$$

where $\boldsymbol{R}^{2} \psi$ is the unique solution in $V^{2}$, of the problem:

$$
a^{2}\left(\boldsymbol{R}^{2}(\psi), \boldsymbol{v}\right)=\langle\psi, \boldsymbol{v}\rangle_{2} \quad \forall \boldsymbol{v} \in V^{2}
$$

Let us consider the following norm in $V^{2^{\prime}}$ :

$$
\|\psi\|=\left(a^{2}\left(\boldsymbol{R}^{2} \psi, \boldsymbol{R}^{2} \psi\right)\right)^{\frac{1}{2}}
$$

Proposition 5.5. $\|\cdot\|$ and $\|\cdot\|_{V^{2^{\prime}}}$ are equivalent norms on the space $V^{2}$.

Proof. By using $\boldsymbol{v}=\boldsymbol{R}^{2}(\psi)$ as test function in (5.20), the proof is the direct consequence of (5.21) and of the continuity and the coerciveness of $a^{2}(\cdot, \cdot)$, so that we get:

$$
\begin{gathered}
\|\psi\| \leq \frac{1}{\sqrt{m}}\|\psi\|_{V^{2^{\prime}}}, \\
\frac{M}{\sqrt{m}}\|\psi\| \geq\|\psi\|_{V^{2^{\prime}}} .
\end{gathered}
$$


Proposition 5.6. There exist constants $C_{i}, i=0, \ldots, 5$, such that $T_{\theta}$ is a contraction for any $\theta$ in

$$
\begin{array}{ll}
] C_{0}, C_{1}\left[\quad \text { if } \| k \| _ { L ^ { \infty } ( \Gamma _ { c } ) } \in \left[0, C_{2}[.\right.\right. \\
] 0, C_{0}\left[\quad \text { if } \| k \| _ { L ^ { \infty } ( \Gamma _ { c } ) } \in \left[0, C_{3}[.\right.\right. \\
] 0, C_{0}\left[\quad \text { if }\|k\|_{L^{\infty}\left(\Gamma_{c}\right)} \in\right] C_{3}, C_{4}[. \\
] C_{6}, C_{0}\left[\quad \text { if }\|k\|_{L^{\infty}\left(\Gamma_{c}\right)} \in\right] C_{4}, C_{5}[.
\end{array}
$$

Proof. Let $\psi$ and $\tilde{\psi}$ in $V^{2^{\prime}}, \boldsymbol{w}^{1}$ (resp. $\tilde{\boldsymbol{w}}^{1}$ ) and $\boldsymbol{w}^{2}$ (resp. $\tilde{\boldsymbol{w}}^{2}$ ) be the solutions of (5.5) and (5.6). From the definition of $T_{\theta}$ we have:

$$
\left\|T_{\theta}(\psi)-T_{\theta}(\tilde{\psi})\right\|^{2}=\theta^{2}\|T(\psi)-T(\tilde{\psi})\|^{2}+(1-\theta)^{2}\|\psi-\tilde{\psi}\|^{2}+2 \theta(1-\theta) a^{2}\left(\boldsymbol{R}^{2}(T(\psi)-T(\tilde{\psi})), \boldsymbol{R}^{2}(\psi-\tilde{\psi})\right) .
$$

To prove that the operator $T_{\theta}$ is a contraction, we give, in the first step, an estimate for the term $a^{2}\left(\boldsymbol{R}^{2}(T(\psi)-T(\tilde{\psi})), \boldsymbol{R}^{2}(\psi-\tilde{\psi})\right)$ in terms of $\|T(\psi)-T(\tilde{\psi})\|$ and $\|\psi-\tilde{\psi}\|$. We use the definitions of operators $T$ and $\boldsymbol{R}^{2}$ and the equivalence between $\|\cdot\|$ and $\|\cdot\|_{V^{2^{\prime}}}$.

First step. From the uniqueness of the solution of (5.20), we get $\boldsymbol{R}^{2}(\psi-\tilde{\psi})=\boldsymbol{w}^{2}-\tilde{\boldsymbol{w}}^{2}$ so

$$
a^{2}\left(\boldsymbol{R}^{2}(T(\psi)-T(\tilde{\psi})), \boldsymbol{R}^{2}(\psi-\tilde{\psi})\right)=a^{2}\left(\boldsymbol{R}^{2}(T(\psi)-T(\tilde{\psi})), \boldsymbol{w}^{2}-\tilde{\boldsymbol{w}}^{2}\right),
$$

using the definition (5.20) of $\boldsymbol{R}^{2}$, we have:

$$
a^{2}\left(\boldsymbol{R}^{2}(T(\psi)-T(\tilde{\psi})), \boldsymbol{w}^{2}-\tilde{\boldsymbol{w}}^{2}\right)=\left\langle T \psi-T \tilde{\psi}, \boldsymbol{w}^{2}-\tilde{\boldsymbol{w}}^{2}\right\rangle_{2} .
$$

The definition (5.4) of $T$ gives

$$
\left\langle T \psi-T \tilde{\psi}, \boldsymbol{w}^{2}-\tilde{\boldsymbol{w}}^{2}\right\rangle_{2}=-a^{1}\left(\boldsymbol{w}^{1}-\tilde{\boldsymbol{w}}^{1}, \boldsymbol{R}_{D}^{1} \gamma\left(\boldsymbol{w}^{2}-\tilde{\boldsymbol{w}}^{2}\right)\right) .
$$

From inequality (5.12), we obtain:

$$
\begin{aligned}
-a^{1}\left(\boldsymbol{w}^{1}-\tilde{\boldsymbol{w}}^{1}, \boldsymbol{R}_{D}^{1} \gamma\left(\boldsymbol{w}^{2}-\tilde{\boldsymbol{w}}^{2}\right)\right) \leq & -a^{1}\left(\boldsymbol{w}^{1}-\tilde{\boldsymbol{w}}^{1}, \boldsymbol{w}^{1}-\tilde{\boldsymbol{w}}^{1}\right) \\
& -\int_{\Gamma_{c}} k\left(\left|S\left(\sigma_{N}\left(\boldsymbol{w}^{2}\right)\right)\right|-\left|S\left(\sigma_{N}\left(\tilde{\boldsymbol{w}}^{2}\right)\right)\right|\right)\left(\left|\left[\boldsymbol{w}_{T}\right]\right|-\left|\left[\tilde{\boldsymbol{w}}_{T}\right]\right|\right) .
\end{aligned}
$$

In the right-hand side, the first term will be estimated by using the coerciveness of $a^{1}(.,$.$) , inequalities (5.18)$ and (5.22):

$$
-a^{1}\left(\boldsymbol{w}^{1}-\tilde{\boldsymbol{w}}^{1}, \boldsymbol{w}^{1}-\tilde{\boldsymbol{w}}^{1}\right) \leq-\frac{m^{2}}{M^{2} C_{R}^{2}}\|T(\psi)-T(\tilde{\psi})\|^{2}
$$

for the second term, using inequalities (5.11) (see Lem. 5.3) and (5.19) in (5.13) and using (5.23), we obtain:

$$
-\int_{\Gamma_{c}} k\left(\left|S\left(\sigma_{N}\left(\boldsymbol{w}^{2}\right)\right)\right|-\left|S\left(\sigma_{N}\left(\tilde{\boldsymbol{w}}^{2}\right)\right)\right|\right)\left(\left|\left[\boldsymbol{w}_{T}\right]\right|-\left|\left[\tilde{\boldsymbol{w}}_{T}\right]\right|\right) \leq \frac{C_{K}}{m^{2}}\left(M C_{R}+C_{K}+\sqrt{C_{K} m}+1\right) \frac{M^{2}}{m}\|\psi-\tilde{\psi}\|^{2} .
$$

Taking inequalities (5.26)-(5.30) into account in (5.25), we obtain:

$$
\begin{aligned}
a^{2}\left(\boldsymbol{R}^{2}(T(\psi)-T(\tilde{\psi})), \boldsymbol{R}^{2}(\psi-\tilde{\psi})\right) \leq & -\frac{m^{2}}{M^{2} C_{R}^{2}}\|T(\psi)-T(\tilde{\psi})\|^{2} \\
& +\frac{C_{K}}{m^{2}}\left(M C_{R}+C_{K}+\sqrt{C_{K} m}+1\right) \frac{M^{2}}{m}\|\psi-\tilde{\psi}\|^{2} .
\end{aligned}
$$


Using (5.31) and (5.24), we obtain:

$$
\begin{aligned}
\left\|T_{\theta}(\psi)-T_{\theta}(\tilde{\psi})\right\|^{2} \leq\left(\theta^{2}-\frac{m^{2} 2 \theta(1-\theta)}{M^{2} C_{R}^{2}}\right)\|T(\psi)-T(\tilde{\psi})\|^{2} & \\
& +\left((1-\theta)^{2}+2 \theta(1-\theta) \frac{C_{K}}{m^{2}}\left(M C_{R}+C_{K}+\sqrt{C_{K} m}+1\right) \frac{M^{2}}{m}\right)\|\psi-\tilde{\psi}\|^{2} .
\end{aligned}
$$

Using (5.16), we get:

$$
\begin{aligned}
\left\|T_{\theta}(\psi)-T_{\theta}(\tilde{\psi})\right\|^{2} \leq\left(\theta^{2}-\frac{m^{2} 2 \theta(1-\theta)}{M^{2} C_{R}^{2}}\right) \| & T(\psi)-T(\tilde{\psi}) \|^{2} \\
& +\left((1-\theta)^{2}+2 \theta(1-\theta) \frac{M^{2}}{m}\left(\frac{C_{K} C_{T}}{M C_{R}}+\frac{C_{K}}{m^{2}}\right)\right)\|\psi-\tilde{\psi}\|^{2} .
\end{aligned}
$$

Second step. Later on, it is convenient to introduce:

$$
\tilde{m}=\min \{m, 1\}, \quad \tilde{M}=M .
$$

We immediately have from (5.16)

$$
C_{T} \leq \tilde{C}_{T}=\frac{\tilde{M} C_{R}}{\tilde{m}^{2}}\left(\tilde{M} C_{R}+C_{K}+\sqrt{C_{K} \tilde{m}}\right)
$$

Inequality (5.33) becomes:

$$
\begin{aligned}
\left\|T_{\theta}(\psi)-T_{\theta}(\tilde{\psi})\right\|^{2} \leq\left(\theta^{2}-\frac{\tilde{m}^{2} 2 \theta(1-\theta)}{\tilde{M}^{2} C_{R}^{2}}\right) \| & T(\psi)-T(\tilde{\psi}) \|^{2} \\
& +\left((1-\theta)^{2}+2 \theta(1-\theta) \frac{\tilde{M}^{2}}{\tilde{m}}\left(\frac{C_{K} \tilde{C}_{T}}{\tilde{M} C_{R}}+\frac{C_{K}}{\tilde{m}^{2}}\right)\right)\|\psi-\tilde{\psi}\|^{2} .
\end{aligned}
$$

According to the sign of the coefficient of $\|T(\psi)-T(\tilde{\psi})\|^{2}$, we distinguish two cases: in the first one, the Lipschitz continuity of the operator $T$ is used, and in the second one, we only have to cancel this term:

\section{Case 5.7.}

$$
\text { For any } \theta \geq C_{0} \quad \text { with } \quad C_{0}=\frac{2 \tilde{m}^{2}}{\tilde{M}^{2} C_{R}^{2}+2 \tilde{m}^{2}}
$$

we have $\theta^{2}-\frac{\tilde{m}^{2} 2 \theta(1-\theta)}{\tilde{M}^{2} C_{R}^{2}} \geq 0$, so that Proposition 5.4 implies

$$
\left(\theta^{2}-\frac{\tilde{m}^{2} 2 \theta(1-\theta)}{\tilde{M}^{2} C_{R}^{2}}\right)\|T(\psi)-T(\tilde{\psi})\|^{2} \leq\left(\theta^{2}-\frac{\tilde{m}^{2} 2 \theta(1-\theta)}{\tilde{M}^{2} C_{R}^{2}}\right) C_{T}^{2} \frac{\tilde{M}^{2}}{\tilde{m}^{2}}\|\psi-\tilde{\psi}\|^{2}
$$

Hence, inequality (5.36) becomes

$$
\left\|T_{\theta}(\psi)-T_{\theta}(\tilde{\psi})\right\|^{2} \leq h_{1}(\theta)\|\psi-\tilde{\psi}\|^{2}
$$


with

$$
\begin{aligned}
h_{1}(\theta) & =\left(\theta^{2}-\frac{\tilde{m}^{2} 2 \theta(1-\theta)}{\tilde{M}^{2} C_{R}^{2}}\right) C_{T}^{2} \frac{\tilde{M}^{2}}{\tilde{m}^{2}}+(1-\theta)^{2}+2 \theta(1-\theta) \frac{\tilde{M}^{2}}{\tilde{m}}\left(\frac{C_{K} \tilde{C}_{T}}{\tilde{M} C_{R}}+\frac{C_{K}}{\tilde{m}^{2}}\right) \\
& =a \theta^{2}+b \theta+c,
\end{aligned}
$$

in which

$$
\begin{aligned}
& a=\tilde{C}_{T} \frac{\tilde{M}^{2}}{\tilde{m}^{2}}+\frac{2{\tilde{C_{T}}}^{2}}{C_{R}^{2}}-2\left(\frac{C_{K} \tilde{C_{T}}}{\tilde{M} C_{R}}+\frac{C_{K}}{\tilde{m}^{2}}\right) \frac{\tilde{M}^{2}}{\tilde{m}}+1 \\
& b=-\frac{2 \tilde{C}_{T}^{2}}{C_{R}^{2}}+2\left(\frac{C_{K}}{\tilde{M} C_{R}}+\frac{C_{K}}{\tilde{m}^{2}}\right) \frac{\tilde{M}^{2}}{\tilde{m}}-2 \\
& c=1 .
\end{aligned}
$$

In the following, we will prove first that $a>0$ and $b<0$, so that $h_{1}(\theta)$ will be less than 1 for any $\theta$ in $] 0,-\frac{b}{a}[$. As $\theta$ belongs to $\left[C_{0}, 1[(c f .(5.37))\right.$, we will prove under some conditions for the friction coefficient that ] $0,-\frac{b}{a}\left[\cap\left[C_{0}, 1\left[\right.\right.\right.$ is not empty. This will allow us to obtain an interval of $\theta$ in which $T_{\theta}$ is a contraction.

- From the definition (5.35) of $\tilde{C}_{T}$, we have (as $C_{K} \geq 0$ )

$$
\tilde{C_{T}} \geq \frac{\tilde{M} C_{R} C_{K}}{\tilde{m}^{2}}+\frac{\tilde{M} C_{R} \sqrt{C_{K} \tilde{m}}}{\tilde{m}^{2}}
$$

and

$$
\tilde{C_{T}} \geq \frac{\tilde{M} C_{R} \sqrt{C_{K} \tilde{m}}}{\tilde{m}^{2}}
$$

so

$$
\tilde{C}_{T}^{2} \geq \frac{\tilde{M} C_{R} C_{K} \tilde{C}_{T}}{\tilde{m}^{2}}+\frac{\tilde{M}^{2} C_{R}^{2} C_{K}}{\tilde{m}^{3}}
$$

As $\tilde{m} \leq 1(c f .(5.34))$, we get:

$$
\frac{\tilde{C}_{T}^{2}}{C_{R}^{2}} \geq \frac{\tilde{M} C_{K} \tilde{C}_{T}}{\tilde{m} C_{R}}+\frac{\tilde{M}^{2} C_{K}}{\tilde{m}^{3}}
$$

From the above expression of $a$, we get that $a>0$ and $b<0$, more precisely $a \geq 1$ and $b \leq-2$.

- Let

$$
C_{1}=-\frac{b}{a}
$$

As mentioned above, the contraction of $T_{\theta}$ is proved as soon as $\left.\theta \in\right] 0, C_{1}\left[\cap\left[C_{0}, 1\left[\right.\right.\right.$ if $C_{0}<C_{1}$. 
Using the fact that $a={\tilde{C_{T}}}^{2} \frac{\tilde{M}^{2}}{\tilde{m}^{2}}-b-1$, we have

$$
\begin{aligned}
C_{0}-C_{1} & =\frac{2 \tilde{m}^{2}}{\tilde{M}^{2} C_{R}^{2}+2 \tilde{m}^{2}}+\frac{b}{\tilde{C}_{T}^{2} \frac{\tilde{M}^{2}}{\tilde{m}^{2}}-b-1} \\
& =\frac{2 \tilde{C}_{T}^{2} \tilde{M}^{2}+b \tilde{M}^{2} C_{R}^{2}-2 \tilde{m}^{2}}{\left(\tilde{M}^{2} C_{R}^{2}+2 \tilde{m}^{2}\right) a} \\
& =\frac{-2 \tilde{m}^{2}+2\left(\frac{C_{K} \tilde{C}_{T}}{\tilde{M} C_{R}}+\frac{C_{K}}{\tilde{m}^{2}}\right) \frac{\tilde{M}^{4} C_{R}^{2}}{\tilde{m}}-2 \tilde{M}^{2} C_{R}^{2}}{\left(\tilde{M}^{2} C_{R}^{2}+2 \tilde{m}^{2}\right) a} \\
& =\frac{-2 \tilde{m}^{2}-2 \tilde{M}^{2} C_{R}^{2}+\frac{2 \tilde{M}^{4} C_{R}^{2}}{\tilde{m}^{3}}\left(C_{K}^{2}+\sqrt{\tilde{m}} C_{K}^{\frac{3}{2}}+\left(\tilde{M} C_{R}+1\right) C_{K}\right)}{\left(\tilde{M}^{2} C_{R}^{2}+2 \tilde{m}^{2}\right) a} .
\end{aligned}
$$

Let

$$
\mathcal{G}(x)=\tilde{M}^{2}\left(x^{2}+\sqrt{\tilde{m}} x^{\frac{3}{2}}+\left(\tilde{M} C_{R}+1\right) x\right) \quad \forall x \geq 0,
$$

so from (5.41), we have:

$$
C_{0}<C_{1} \Leftrightarrow-2 \tilde{m}^{2}-2 \tilde{M}^{2} C_{R}^{2}+\frac{2 \tilde{M}^{2} C_{R}^{2}}{\tilde{m}^{3}} \mathcal{G}\left(C_{K}\right)<0 .
$$

Using the fact that $\mathcal{G}$ is a strictly increasing function from $[0,+\infty[$, the previous inequality (5.43) is equivalent to:

$$
C_{K} \in\left[0, \mathcal{G}^{-1}\left(\frac{\tilde{m}^{5}}{\tilde{M}^{2} C_{R}^{2}}+\tilde{m}^{3}\right)[\right.
$$

which is equivalent to:

$$
\|k\|_{L^{\infty}\left(\Gamma_{c}\right)} \in\left[0, C_{2}\left[, \quad \text { with } \quad C_{2}=\frac{\mathcal{G}^{-1}\left(\frac{\tilde{m}^{5}}{\tilde{M}^{2} C_{R}^{2}}+\tilde{m}^{3}\right)}{C_{s} C_{t r}}\right.\right.
$$

The first result of Proposition 5.6 is then proved.

Case 5.8. If $\theta<C_{0}$, inequality (5.33) becomes:

$$
\left\|T_{\theta}(\psi)-T_{\theta}(\tilde{\psi})\right\|^{2} \leq h_{2}(\theta)\|\psi-\tilde{\psi}\|^{2}
$$

in which:

$$
\begin{aligned}
h_{2}(\theta) & =(1-\theta)^{2}+2 \theta(1-\theta) \frac{\tilde{M}^{2}}{\tilde{m}}\left(\frac{C_{K} \tilde{C}_{T}}{\tilde{M} C_{R}}+\frac{C_{K}}{\tilde{m}^{2}}\right) \\
& =a^{\prime} \theta^{2}+b^{\prime} \theta+1,
\end{aligned}
$$

with

$$
\begin{aligned}
& a^{\prime}=1-2 \frac{\tilde{M}^{2}}{\tilde{m}}\left(\frac{C_{K} \tilde{C}_{T}}{\tilde{M} C_{R}}+\frac{C_{K}}{\tilde{m}^{2}}\right) \\
& b^{\prime}=-2+2 \frac{\tilde{M}^{2}}{\tilde{m}}\left(\frac{C_{K} \tilde{C}_{T}}{\tilde{M} C_{R}}+\frac{C_{K}}{\tilde{m}^{2}}\right) .
\end{aligned}
$$




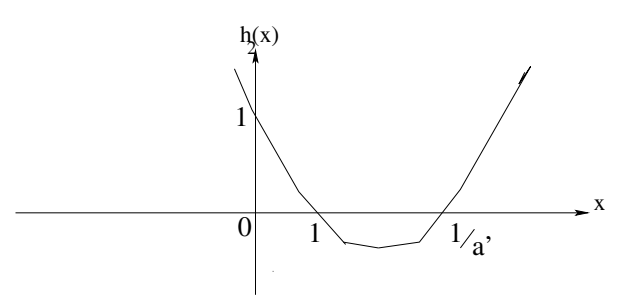

Figure 1. Case: $a^{\prime}>0$.



Figure 2. Case: $-1<a^{\prime}<0$.

As $a^{\prime}=-b^{\prime}-1$, we have

$$
h_{2}(\theta)=a^{\prime}(\theta-1)\left(\theta-\frac{1}{a^{\prime}}\right) .
$$

Case 2-1: $a^{\prime}>0$.

In this case, we have from the expression of $a^{\prime}, a^{\prime}<1$, so

$$
\frac{1}{a^{\prime}}>1 \text {. }
$$

Following the representation of $h_{2}$ in Figure 1, we have $0<h_{2}(\theta)<1$ for any $\theta$ in $] 0,1\left[\cap\left[0, C_{0}[=] 0, C_{0}[\right.\right.$.

Let us characterize the positive sign of $a^{\prime}$ by a condition for the friction coefficient. Using the notation (5.42), we have:

$$
a^{\prime}=1-\frac{2}{\tilde{m}^{3}} \mathcal{G}\left(C_{K}\right)
$$

By simple calculations, we see by using (5.42) that:

$$
a^{\prime}>0 \Longleftrightarrow 0 \leq C_{K}<\mathcal{G}^{-1}\left(\frac{\tilde{m}^{3}}{2}\right)
$$

By taking $C_{3}=\frac{\mathcal{G}^{-1}\left(\frac{\tilde{m}^{3}}{2}\right)}{C_{s} C_{t r}}$, the second wording of Proposition 5.6 is obtained.

As $h_{2}\left(1+\frac{1}{a^{\prime}}\right)=1$, we have in the case: $a^{\prime}<0$ two possibilities following the sign of $1+\frac{1}{a^{\prime}}$.

Case 2-2: $-1<a^{\prime}<0$.

In this case, the representation of $h_{2}$ is given in Figure 2 and $0<h_{2}(\theta)<1$, for any $\theta$ in $] 0,1\left[\cap\left[0, C_{0}[=] 0, C_{0}[\right.\right.$.

By using (5.46), the condition $-1<a^{\prime}<0$ is equivalent to $\mathcal{G}^{-1}\left(\frac{\tilde{m}^{3}}{2}\right)<C_{K}<\mathcal{G}^{-1}\left(\tilde{m}^{3}\right)$. The third wording of Proposition 5.6 is obtained by taking

$$
C_{4}=\frac{\mathcal{G}^{-1}\left(\tilde{m}^{3}\right)}{C_{s} C_{t r}}
$$




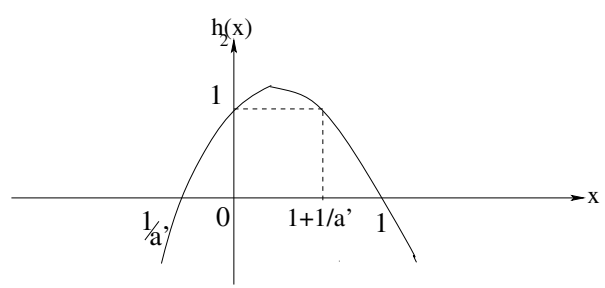

Figure 3. Case: $a^{\prime}<-1$.

Case 2-3: $a^{\prime}<-1$.

From the representation of $h_{2}$ in Figure 3, we have $0<h_{2}(\theta)<1$ for any $\theta$ in $] 1+\frac{1}{a^{\prime}}, 1\left[\cap\left[0, C_{0}[=] 1+\frac{1}{a^{\prime}}, C_{0}[\right.\right.$ if $1+\frac{1}{a^{\prime}}<C_{0}$.

The two conditions: $a^{\prime}<-1$ and $1+\frac{1}{a^{\prime}}<C_{0}$ are equivalent to: $\mathcal{G}^{-1}\left(\tilde{m}^{3}\right)<C_{K}<\mathcal{G}^{-1}\left(\frac{2-C_{0}}{1-C_{0}} \frac{\tilde{m}^{3}}{2}\right)$.

The last result of Proposition 5.6 is proved by taking

$$
\begin{aligned}
C_{5} & =\frac{\mathcal{G}^{-1}\left(\frac{2-C_{0}}{1-C_{0}} \frac{\tilde{m}^{3}}{2}\right)}{C_{s} C_{t r}} \\
C_{6} & =1+\frac{1}{a^{\prime}} .
\end{aligned}
$$

Theorem 5.9. Assuming that the relaxation parameter $\theta$ and the coefficient of friction $k$ satisfy Proposition 5.6 , then the sequence $\left(g_{i}^{2}\right)_{i \geq 0}$ converges in $V^{2^{\prime}}$ towards $g^{2}$ defined by:

$$
\left\langle g^{2}, \boldsymbol{v}\right\rangle_{2}=-a^{1}\left(\boldsymbol{u}^{1}, \boldsymbol{R}_{D}^{1}(\gamma \boldsymbol{v})\right)+L^{1}\left(\boldsymbol{R}_{D}^{1}(\gamma \boldsymbol{v})\right) \quad \forall \boldsymbol{v} \in V^{2}
$$

where $\left(\boldsymbol{u}^{1}, \boldsymbol{u}^{2}\right)$ is the solution of the problem $\left(P_{1}\right)$.

Moreover, if $\theta \geq \theta_{\min }$, the solution $\boldsymbol{u}_{i}^{\alpha}, \alpha=1,2$ of the algorithm (4.9) converges to the solution $\boldsymbol{u}=\left(\boldsymbol{u}^{1}, \boldsymbol{u}^{2}\right)$ of the problem $\left(P_{1}\right)$ defined in (4.4) as i tends to infinity.

Proof. From algorithm $\left(Q_{i}\right)$, it is easy to prove that $\left\langle T g_{i-1}^{2}, \boldsymbol{v}\right\rangle_{2}=-a^{1}\left(\boldsymbol{u}_{i}^{1}, \boldsymbol{R}_{D}^{1}(\gamma \boldsymbol{v})\right)+L^{1}\left(\boldsymbol{R}_{D}^{1}(\gamma \boldsymbol{v})\right)$ $\forall \boldsymbol{v} \in V^{2}$, so $T_{\theta}\left(g_{i-1}^{2}\right)=g_{i}^{2}$.

On the other hand, one deduces from problems $\left(P_{2}\right)$ and $\left(P_{3}\right)$ that $T_{\theta}\left(g^{2}\right)=g^{2}$. Hence from Proposition 5.6 we have:

$$
\left\|g_{i}^{2}-g^{2}\right\|=\left\|T_{\theta}\left(g_{i-1}^{2}\right)-T_{\theta}\left(g^{2}\right)\right\| \leq K(\theta)\left\|g_{i-1}^{2}-g^{2}\right\| .
$$

The convergence of $g_{i}^{2}$ is obtained by a recursive application of (5.47).

Proposition 5.1 concludes the proof.

Remark 5.10. Assumptions of Theorem 5.9 are fulfilled for the Signorini problem $(k=0)$ in which, only the unilateral contact is taken into account.

\section{Numerical RESULtS}

As mentioned in the introduction, a Neumann-Dirichlet algorithm for contact problem with Coulomb friction has already been used in $[14,16]$ in a variety of situations. The aim of this section is to illustrate and validate the previous theoretical approach for a large contact device, especially with respect of the choice of the relaxation parameter.

The implementation is established using the finite element code PLAST2 developed in Laboratoire de Mécanique des Contact et des Solides LaMCoS of the National Institute of Applied Sciences in Lyon. 


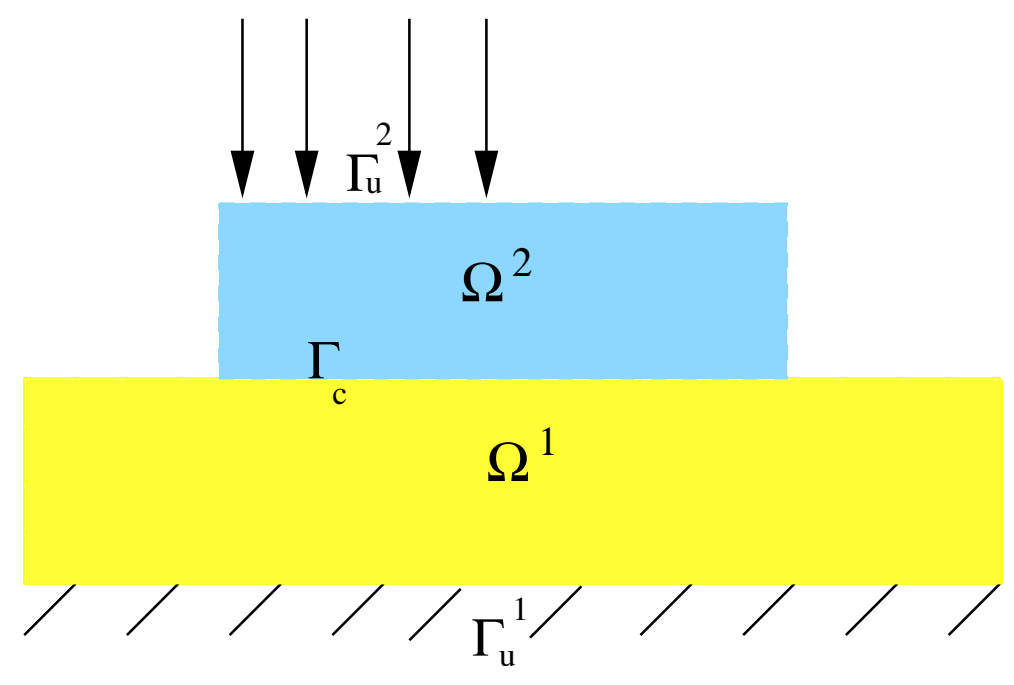

Figure 4. The physical problem.

Based on the Lagrange multiplier concept [3], PLAST2 is generally used for solving contact problems by global method with nonmatching grids at the contact zone (see [2]). The Neumann-Dirichlet algorithm described in the previous section was implemented in PLAST2. In each step of our algorithm, we solve two local problems: a linear Neumann problem on the first body and a contact problem with given friction on the second body.

After describing the physical data, we compare the interface coordinates and the contact stresses calculated by the Neumann-Dirichlet method to the results given by the global method using the PLAST2 code. Then, several results concerning the influence of the coefficient friction, the Young modulus, the mesh-size on the convergence rate are given and the existence of an optimal relaxation parameter is shown.

\subsection{Description of the model}

For all experiments described below, a two dimensional linear elasticity problem of two elastic bodies $\Omega^{\alpha}$, $\alpha=1,2$ initially in contact is considered (see Fig. 4). The elastic behavior law is given by Hooke's law for homogeneous isotropic elastic materials:

$$
\sigma_{i j}^{\alpha}(\boldsymbol{u})=\frac{E_{\alpha} \nu_{\alpha}}{\left(1-2 \nu_{\alpha}\right)\left(1+\nu_{\alpha}\right)} \delta_{i j} \varepsilon_{k k}(\boldsymbol{u})+\frac{E_{\alpha}}{1+\nu_{\alpha}} \varepsilon_{i j}(\boldsymbol{u}), \quad \alpha=1,2,
$$

where $E_{\alpha}$ and $\nu_{\alpha}$ denote Young modulus and Poisson's ratio respectively for the body $\Omega^{\alpha}, \alpha=1,2$, and the notation $\delta_{i j}$ stands for Kronecher's symbol.

The lower body $\Omega^{1}$ (the slave) is fixed on its lower side. The upper body $\Omega^{2}$ (the master) is submitted to a vertical displacement of $-0.015 \mathrm{~mm}$ on $\Gamma_{u}^{2}$ (see Fig. 4). Dimensions of $\Omega^{1}$ and $\Omega^{2}$ are respectively $2 \mathrm{~mm} \times 0.3 \mathrm{~mm}$ and $1.2 \mathrm{~mm} \times 0.3 \mathrm{~mm}$.

\subsection{Preliminary results}

In the first sequence of computations, we use the following data: for both solids, the Poisson's ratio is $\nu_{1}=\nu_{2}=0.3$. The Young modulus $E_{1}=7 \times 10^{4} \mathrm{MPa}$ for the lower body and $E_{2}=2 \times 10^{4} \mathrm{MPa}$ for the upper body are assumed. We use $k=0.2$ as friction coefficient and a mesh-size of 190 nodes (see Fig. 7).

Figure 5 depicts the coordinates of the points of the contact interface (after deformation) and the values of the normal and tangential constraints obtained by the Neumann-Dirichlet methods. The horizontal axis in Figure 5(a) is the classical $x$ coordinates with $x=0$ at the left side of the contact interface. 


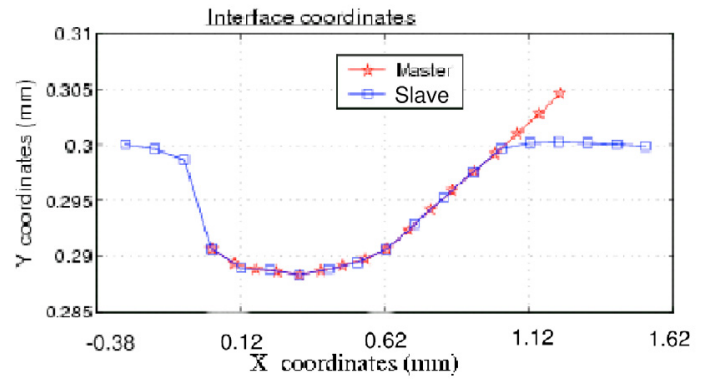

(a)

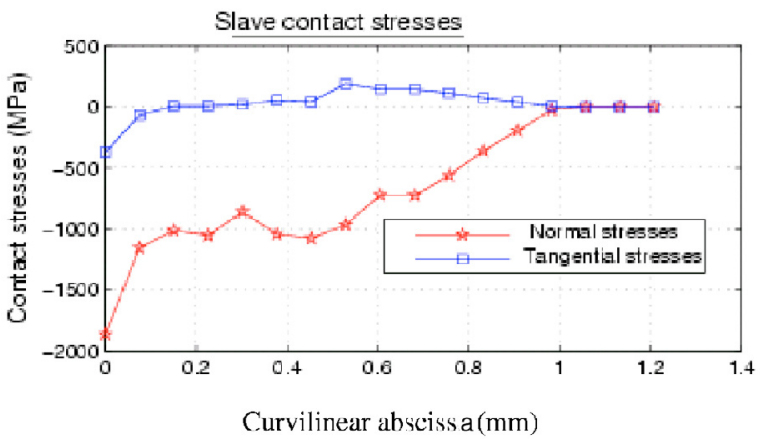

(b)

Figure 5. Interface coordinates and contact stresses by the Neumann-Dirichlet method.

In Figure 5(b), the horizontal axis is the curvilinear abscissa for the deformed slave contact interface measured from the same origin as the $x$ coordinate. These figures evidence that the contact zone is represented by three parts; a sliding one located for $0<x \leq 0.6$, the slippery one located for $0.6<x<1$, while the non contact area is located for $x>1$.

To be mentioned that we find exactly the same results by using the classical method (without decomposition).

\subsection{About the optimum relaxation parameter}

In the following, we present numerical results which shown the influence of the friction coefficient, the Young modulus and the mesh-size on the number of iterations in the Neumann-Dirichlet algorithm. The stopping criteria is defined by: $\left\|g_{n}^{2}-g_{n-1}^{2}\right\|_{2} /\left\|g_{n}^{2}\right\|_{2}<10^{-9}$ or $\left\|g_{n}^{2}-g_{n-1}^{2}\right\|_{2}<10^{-9}$.

We present in Figure 10, the number of iterations as a function of the parameter $\theta$ for 4 mesh-size with $k$, $E 1, E 2$, and $\nu$ fixed $\left(k=0.2, E 1=0.7 \times 10^{5}, E 2=0.2 \times 10^{5}, \nu=0.3\right)$. The mesh size varies according the number of nodes from 60 nodes to 2482 nodes (see Figs. 6-9).

It clearly appears from (Fig. 10) that the number of iterations is not strongly dependent to the mesh-size. Especially there exists an optimum value of relaxation parameters $\left(\theta_{\mathrm{opt}}=0.6\right)$ which is nearly independent of the choice of the mesh-size. The result is important as this will be sufficient to find the optimal parameter by solving small dimension problems first.

Varying the value of $k, k=0.1,0.2,0.4$ (Fig. 11) does not modify the optimal value of the relaxation parameter which is always located around 0.6 with $E 1 E 2, \nu$ and a mesh-size (190 nodes) fixed.

Finally, choosing the first body much harder $\left(E 1=0.7 \times 10^{5}, E 2=0.2 \times 10^{5}\right)$ or much softer than the second body $\left(E 1=0.2 \times 10^{5}, E 2=0.7 \times 10^{5}\right)$ or choosing the same Young modulus $\left(E 1=0.2 \times 10^{3}, E 2=0.2 \times 10^{3}\right)$, we obtain the same kind of results (Fig. 12). 




Figure 6. Mesh 1: 60 nodes.

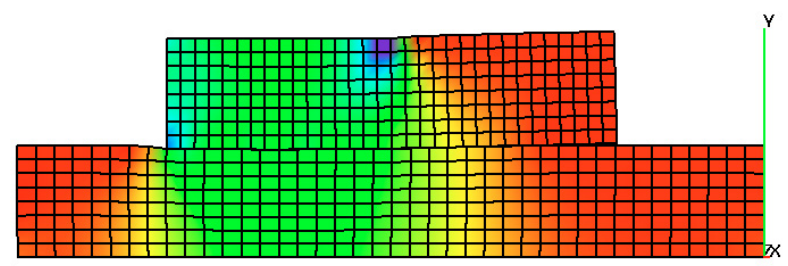

Figure 8. Mesh 3: 666 nodes.



Figure 7. Mesh 2: 190 nodes.

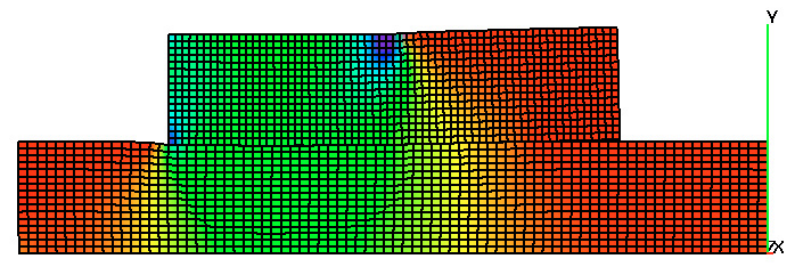

Figure 9. Mesh 4: 2482 nodes.

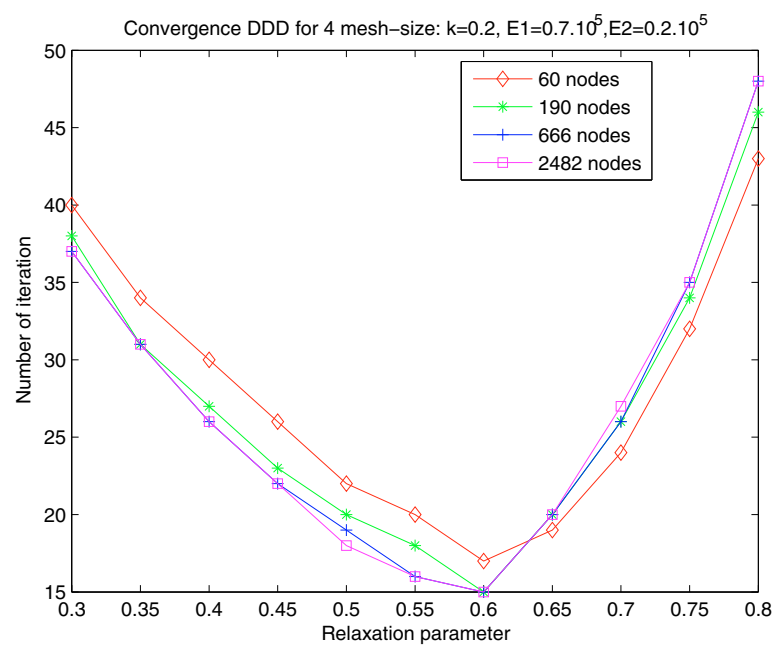

FIGURE 10. Number of iterations as a function of the relaxation parameter $\theta$ for 4 mesh-size with $k=0.2, E 1=0.7 \times 10^{5}, E 2=0.2 \times 10^{5}, \nu=0.3$.

\section{Conclusion}

A Neumann-Dirichlet algorithm of a domain decomposed contact problem with friction has been introduced and studied. For small friction coefficient, convergence result is derived for the continuous problem. The optimal parameter has been numerically found to be independent of several physical and numerical parameters. This phenomena needs further theoretical investigation. The extension of such approach to $2 \mathrm{D}$ and $3 \mathrm{D}$ contact problem between more than 2 bodies should be investigated. 


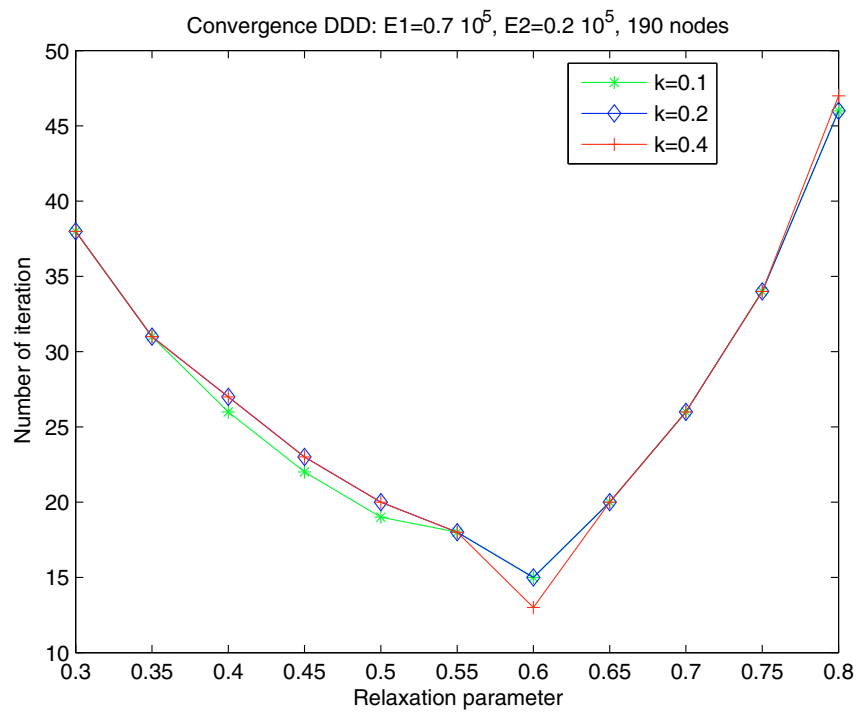

Figure 11. Number of iterations as a function of the relaxation parameter $\theta$ for 3 friction coefficient with $E 1=0.7 \times 10^{5}, E 2=0.2 \times 10^{5}, \nu=0.3,190$ nodes.

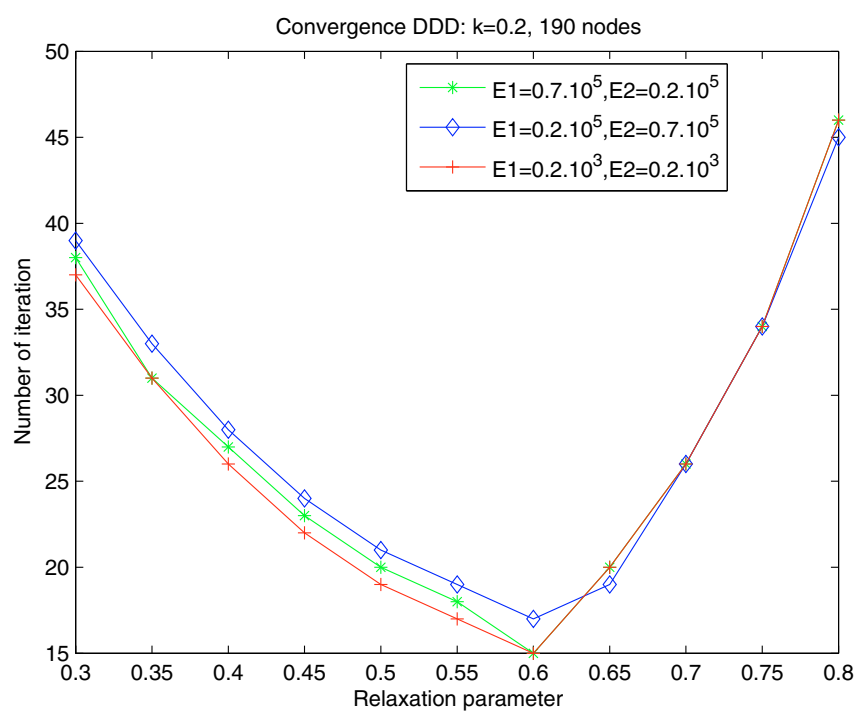

Figure 12. Number of iterations as a function of the relaxation parameter $\theta$ for 3 Young modulus with $k=0.2, \nu=0.3,190$ nodes. 
Acknowledgements. Special thanks to L. Baillet from Laboratoire LaMCos, UMR 5514, INSA de Lyon for his valuable help in the numerical part.

\section{REFERENCES}

[1] P. Alart, M. Barboteu, P. Le Tallec and M. Vidrascu, Méthode de Schwarz additive avec solveur grossier pour problèmes non symétriques. C. R. Acad. Sci. Paris Sér. I Math. 331 (2000) 399-404.

[2] L. Baillet and T. Sassi, Simulations numériques de différentes méthodes d'éléments finis pour les problèmes contact avec frottement. C. R. Acad. Sci. Paris Sér. II B 331 (2003) 789-796.

[3] L. Baillet and T. Sassi, Mixed finite element method for the Signorini problem with friction. Numer. Methods Partial Differential Equations 22 (2006) 1489-1508.

[4] G. Bayada, J. Sabil and T. Sassi, Algorithme de Neumann-Dirichlet pour des problèmes de contact unilatéral: résultat de convergence. C. R. Math. Acad. Sci. Paris 335 (2002) 381-386.

[5] A.B. Chandhary and K.J. Bathe, A solution method for static and dynamic analysis of three-dimensional contact problems with friction. Comput. Struc. 24 (1986) 855-873.

[6] P.W. Christensen, A. Klarbring, J.S. Pang and N. Strömberg, Formulation and comparison of algorithms for frictional contact problems. Internat. J. Numer. Methods Engrg. 42 (1998) 145-173.

[7] G. Duvaut and J.-L. Lions, Les inéquations en mécanique et en physique, Travaux et Recherches Mathématiques 21. Dunod, Paris (1972).

[8] C. Eck and B. Wohlmuth, Convergence of a contact-Neumann iteration for the solution of two-body contact problems. Math. Models Methods Appl. Sci. 13 (2003) 1103-1118.

[9] C. Farhat and F.X. Roux, Implicit parallel processing in structural mechanics. Computational Mechanics Advances 1 (1994) $1-124$.

[10] R. Glowinski, J.-L. Lions and R. Trémolières, Numerical analysis of variational inequalities, Studies in Mathematics and its Applications 8. North-Holland Publishing Co., Amsterdam (1981). Translated from the French.

[11] J. Haslinger, Z. Dostál and R. Kučera, On a splitting type algorithm for the numerical realization of contact problems with Coulomb friction. Comput. Methods Appl. Mech. Engrg. 191 (2002) 2261-2281.

[12] N. Kikuchi and J.T. Oden, Contact problems in elasticity: a study of variational inequalities and finite element methods, SIAM Studies in Applied Mathematics 8. Society for Industrial and Applied Mathematics (SIAM), Philadelphia, PA (1988).

[13] R. Kornhuber and R. Krause, Adaptive multigrid methods for Signorini's problem in linear elasticity. Comput. Vis. Sci. 4 (2001) 9-20.

[14] R.H. Krause, Monotone multigrid methods for Signorini's problem with friction. Ph.D. thesis, University of Berlin, Germany (2001).

[15] R.H. Krause and B.I. Wohlmuth, Nonconforming domain decomposition techniques for linear elasticity. East-West J. Numer. Math. 8 (2000) 177-206.

[16] R.H. Krause and B.I. Wohlmuth, A Dirichlet-Neumann type algorithm for contact problems with friction. Comput. Vis. Sci. 5 (2002) 139-148.

[17] P. Le Tallec, Domain decomposition methods in computational mechanics. Comput. Mech. Adv. 1 (1994) 121-220.

[18] L. Lusternik and V. Sobolev, Précis d'analyse fonctionnelle. MIR, Moscow (1989).

[19] B.F. Smith, P.E. Bjørstad and W.D. Gropp, Domain decomposition, Parallel multilevel methods for elliptic partial differential equations. Cambridge University Press, Cambridge (1996).

[20] G. Zavarise and P. Wriggers, A superlinear convergent augmented Lagrangian procedure for contact problems. Engrg. Comput. 16 (1999) 88-119. 